\title{
Enhancing Immunization Rates in Two Urban Academic Primary Care Clinics: A Before and After Assessment
}

\author{
Zeeshan Yacoob \\ Christopher Cook \\ Fabiana Kotovicz \\ Jessica J.F. Kram \\ Marianne Klumph \\ Marisa Stanley \\ Paul Hunter \\ Dennis J. Baumgardner
}

Follow this and additional works at: https://aah.org/jpcrr

Part of the Allergy and Immunology Commons, Community Health and Preventive Medicine

Commons, Infectious Disease Commons, Influenza Virus Vaccines Commons, Primary Care Commons, Public Health Education and Promotion Commons, and the Virus Diseases Commons

\section{Recommended Citation}

Yacoob Z, Cook C, Kotovicz F, Kram JJ, Klumph M, Stanley M, Hunter P, Baumgardner DJ. Enhancing immunization rates in two urban academic primary care clinics: a before and after assessment. J Patient Cent Res Rev. 2020;7:47-56. doi: 10.17294/2330-0698.1709

Published quarterly by Midwest-based health system Advocate Aurora Health and indexed in PubMed Central, the Journal of Patient-Centered Research and Reviews (JPCRR) is an open access, peer-reviewed medical journal focused on disseminating scholarly works devoted to improving patient-centered care practices, health outcomes, and the patient experience. 


\title{
Enhancing Immunization Rates in Two Urban Academic Primary Care Clinics: A Before and After Assessment
}

\author{
Zeeshan Yacoob, MD, MPH, ${ }^{1,2}$ Christopher Cook, DO, ${ }^{2}$ Fabiana Kotovicz, MD, ${ }^{1,2}$ Jessica J.F. Kram, MPH, ${ }^{1,2,3}$ \\ Marianne Klumph, MA, ${ }^{2,3}$ Marisa Stanley, MPH, ${ }^{5}$ Paul Hunter, MD, ${ }^{1,4}$ Dennis J. Baumgardner, MD ${ }^{1,2,3}$ \\ ${ }^{1}$ University of Wisconsin School of Medicine and Public Health, Madison, WI; ${ }^{2}$ Department of Family Medicine, \\ Aurora UW Medical Group, Aurora Health Care, Milwaukee, WI; ${ }^{3}$ Center for Urban Population Health, Milwaukee, WI; \\ ${ }^{4}$ City of Milwaukee Health Department, Milwaukee, WI; ${ }^{5}$ Eau Claire City-County Health Department, Eau Claire, WI
}

Purpose Immunization rates in many cities in the United States remain suboptimal compared to Healthy People 2020 Goals and are lower than national averages. This study aimed to determine whether a lecturebased educational intervention targeted at nurses and medical assistants would improve vaccination rates.

Methods We conducted a quality improvement study in two urban academic family medicine clinics serving a predominantly Medicaid patient population as well as a sizable proportion of refugees. The intervention consisted of 3 lectures that were delivered to clinic nurses and medical assistants. Vaccinations in 1689 patients - 872 in the 3-month preintervention period, 817 in the 3-month postintervention period - were analyzed.

Results Following the educational intervention, a statistically significant increase was seen only in human papillomavirus vaccine immunization rates for 13-18-year-olds (from $90.7 \%$ [ $n=54$ ] to $100 \%$ [ $n=45$ ]; $\mathrm{P}=0.036)$. When the results were stratified by clinic, only 1 site showed statistically significant increases in: pneumococcal polysaccharide vaccine (23-valent) for high-risk 19-to-64-year-olds (from $36.4 \%$ [ $n=154$ ] to $47.8 \%$ [ $n=136$ ]; $P=0.049$ ); Haemophilus influenzae type $B$ vaccine for 2-month-to5-year-olds (from 91.1\% [ $\mathrm{n}=112]$ to $97.3 \%$ [n=111]; $\mathrm{P}=0.048$ ); and meningococcal conjugate vaccine (quadrivalent) for $13-18$-year olds (from $85.2 \%$ [ $n=27$ ] to $100 \%$ [n=26]; $P=0.042$ ). No increases were seen for our study's refugee patient population $(n=171)$, and a significant decrease of the second-dose measles, mumps, and rubella vaccine $(P=0.036)$ occurred in this subcohort.

Conclusions Ultimately, this quality improvement study demonstrated that educational interventions alone have a limited impact on increasing immunization rates. (J Patient Cent Res Rev. 2020;7:47-56.)

Keywords education; vaccination; primary health care; quality improvement; refugees

$\mathrm{M}$ any of the Healthy People 2020 goals focus on increasing immunization rates among high-risk populations. Unfortunately, with 2020 upon us, many rates remain suboptimal. In the United States thousands of adults die every year from diseases that could have been prevented by vaccines. ${ }^{1-3}$

Correspondence: Fabiana Kotovicz, MD, Aurora Family Medicine Residency Program, 2801 W. Kinnickinnic River Parkway, \#250, Milwaukee, WI 53215 (fabiana.kotovicz@aurora.org)
Moreover, refugee populations are at an increased risk for vaccine-preventable diseases, as the vast majority relocate from developing countries that lack basic health services, are war-torn, or are impoverished. ${ }^{1-4}$

While both reminder recalls and best practice advisories in the past have shown a lot of promise in regards to increasing immunization rates, they do not necessarily prompt the clinician at the time of the visit., ${ }^{5,6}$ This is not ideal, as individuals from disadvantaged communities tend to have fewer interactions with the health care system and are more likely to have fragmented care. ${ }^{7,8}$ 
On average, primary care physicians address 3 problems per encounter, some of which consume the majority of each encounter's time. ${ }^{9}$ Therefore, help from nurses and medical assistants (MAs) can be an extremely valuable tool to increase vaccination rates. ${ }^{10}$ In past studies, when nurses and MAs have been trained on specific preventive health care topics, including vaccines, their measures have improved. ${ }^{11-14}$

By comparing age-appropriate and comorbidityspecific vaccination rates before and after an educational intervention, our study aimed to determine whether a lecture-based educational intervention targeted at nurses and MAs would improve vaccination rates.

\section{METHODS}

This study was deemed non-human subjects research (ie, quality improvement) by Aurora Health Care's institutional review board, thus oversight was waived.

\section{Patient Population and Chart Review}

Patients seen within a 3-month time period at one of two urban academic family medicine clinics located in a midwestern state were analyzed. The preintervention group presented from July 1, 2017, to September 30, 2017; the postintervention group presented from November 1, 2017, to January 31, 2018. A prestudy analysis looking at the aggregate data of the number and types of vaccines administered during each month of the year showed that these two 3-month windows were the most similar in regards to the average number of vaccinations administered per visit and the types of vaccines administered during those visits.

Any patient who had a clinical encounter with one of 20 current postgraduate-year-two (PGY-2) and postgraduate-year-three (PGY-3) family medicine residents met inclusion criteria for the study. Patients were selected randomly using the statistical software $\mathrm{R}$ for each pre- and postintervention chart review. After the random selection, patients whose last visit within the 3-month time span was with one of the study's investigators were excluded. Before the random selection of charts for the postintervention group, patients included in the preintervention chart review were excluded. This was done in order to avoid counting a patient who had been vaccinated during the preintervention period as being up-to-date in the postintervention period (Figure 1).
PGY-2 + PGY-3 Residents

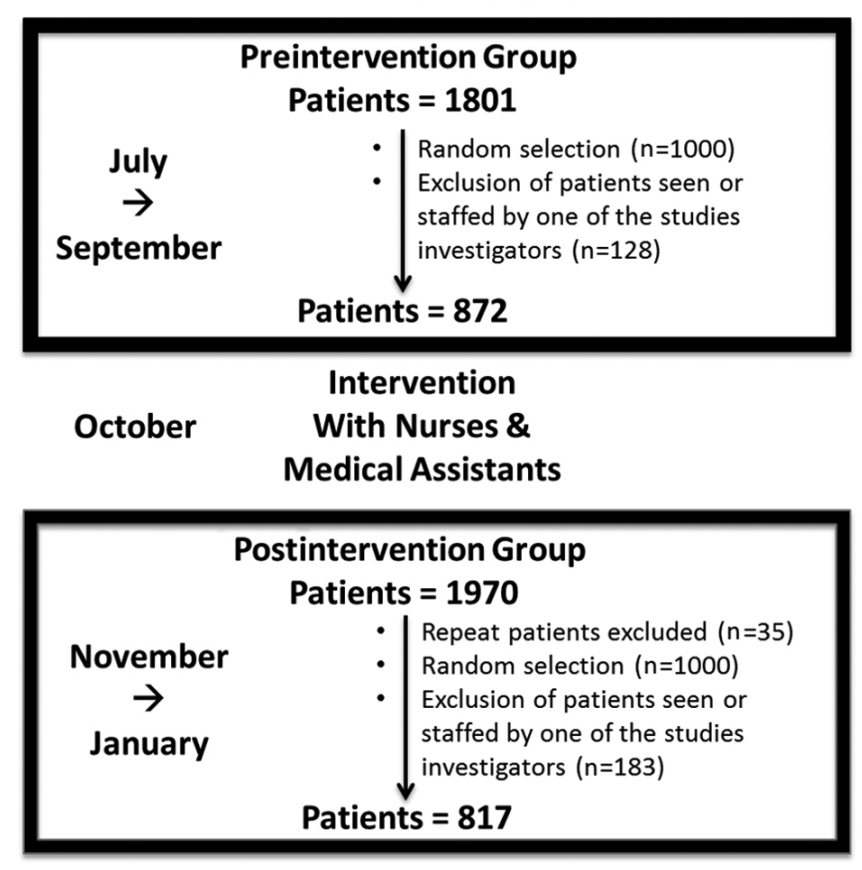

Figure 1. Study timeline and overview of the chart selection process. PGY, postgraduate year.

To determine which vaccine was indicated based on the patient's age and medical comorbidities at the time of the visit, an Excel spreadsheet was coded based on the patient's age and health risks. The encounter date was the most recent date within the 3-month period on which the patient was seen by a PGY-2 or PGY-3 family medicine resident. This date was used to calculate the patient's age in order to determine age-specific vaccine indications.

The coding for vaccine indications was based on the "Recommended Immunization Schedule for Adults Aged 19 Years or Older — United States, 2017" and "Recommended Immunization Schedule for Children and Adolescents Aged 18 Years or Younger — United States, 2017," both of which were released by the Advisory Committee on Immunization Practices and approved by the Centers for Disease Control and Prevention (CDC). ${ }^{15,16}$ For the purpose of this study, vaccines were determined to be up-to-date if the patient had received the complete series for a specific vaccine or if the patient had received the indicated vaccine during their last clinic encounter per documentation in the Wisconsin Immunization Registry or in our health 
system's electronic health record. Definitions for high-risk populations requiring additional vaccines, including patients defined by this study as refugees, are given in Appendix A (p. 55). ${ }^{17-19}$ Abbreviations for vaccines studied in this analysis are provided in Box 1.

For assurance of consistency, a random selection of $40 \%$ of the charts from both pre- and postintervention groups were reviewed a second time by a third investigator. For cases in which a discrepancy was found for a vaccine indication, the study investigators reviewed the chart together to reach a consensus. The occurrence of a discrepancy was very uncommon, and all charts that ultimately required 3-investigator review were deemed to have been done correctly by the primary reviewer.

\section{Intervention}

A case-based lecture series was developed based on standard CDC vaccination recommendations. The lecture series consisted of 3 lectures, each 45 minutes in length, with the goal of addressing immunization guidelines and identified barriers to vaccination. The first lecture covered current vaccination statistics, common misconceptions, and vaccine-preventable diseases. The second lecture covered specific vaccine indications based on age or risk. Lastly, the third lecture covered additional specific vaccine indications, immunization resources, practice recommendations, and communication recommendations. One of the

\begin{tabular}{|c|c|}
\hline \multicolumn{2}{|c|}{ Box 1. Abbreviations for Vaccines } \\
\hline DT & diphtheria and tetanus toxoids \\
\hline DTaP & $\begin{array}{l}\text { diphtheria and tetanus toxoids and } \\
\text { acellular pertussis }\end{array}$ \\
\hline Hep A & hepatitis A \\
\hline Hep B & hepatitis B \\
\hline Hib & Haemophilus influenzae type B \\
\hline HPV & human papillomavirus \\
\hline IPV & inactivated poliovirus \\
\hline MenACWY & meningococcal conjugate, quadrivalent \\
\hline MMR & measles, mumps, and rubella \\
\hline PCV13 & pneumococcal conjugate (13-valent) \\
\hline PPSV23 & pneumococcal polysaccharide (23-valent) \\
\hline Rotavirus & rotavirus vaccine \\
\hline $\mathrm{Td}$ & tetanus and diphtheria \\
\hline Tdap & tetanus, diphtheria, and acellular pertussis \\
\hline Varicella & varicella vaccine \\
\hline
\end{tabular}

investigators, a medical student between his third and fourth year of training, carried out the lectures.

For each clinic site, 3 dates during the month of October 2017 were selected for the educational intervention, in coordination with the clinic manager for both clinics. An email containing the selected dates and inviting all of the nurses and MAs at both sites, with emphasis that participation in the intervention was completely voluntary, was sent.

The intervention took place during the lunch hour. As an incentive, participants were given a $\$ 5$ gift card and light snack for each educational session that they attended. All nurses and MAs who attended the sessions were given a quick reference guide (Appendix B, p. 56 ), created by the study investigators, which included a list of common vaccine indications, the American Academy of Pediatrics "Refusal to Vaccinate" form, and the CDC's 2015 Pneumococcal Vaccine Timing for Adults handout. ${ }^{20,21}$

\section{Statistical Analysis}

Data analysis was conducted using Minitab ${ }^{\circledR}$ statistical software (Minitab, LLC, State College, PA) and Excel (Microsoft Corp., Redmond, WA). All continuous variables were summarized using descriptive statistics, including number (n), mean, standard deviation (SD), median, maximum, and minimum. Analysis of variance (ANOVA) was used for multivariate modeling. Results were considered significantly different if the p-value was less than or equal to 0.05 .

\section{RESULTS}

Vaccinations were reviewed for 1689 randomly selected patients (872 preintervention, 817 postintervention) who met the inclusion criteria (Figure 1). At Clinic 1, all 6 nurses and MAs who were invited attended all 3 education sessions. At Clinic 2, out of the 13 participants who were invited, 5 attended all 3 sessions, 4 attended 2 sessions, and 4 attended 1 session. At both clinics, the vast majority of patients were insured by Medicaid ( $\mathrm{n}=1053,62.3 \%)$, followed by private insurance $(\mathrm{n}=312,18.5 \%)$, and Medicare $(n=286,16.9 \%)$. Overall, the sample population was representative of the patient population at the clinics in terms of age distribution (patients 19-64 years of age: sample $=54.9 \%$; clinic population $=52.3 \%$ ), gender (females: sample $=60.7 \%$; clinic population $=60.0 \%$ ), 
and primary language (English: sample $=79.8 \%$; clinic population $=81.7 \%$ ).

The most common vaccination indication was heart/ lung disease, seen in $37.1 \%(n=626)$ of patients. Additionally, $16.9 \%(\mathrm{n}=285)$ of patients suffered from diabetes and $12.7 \%(n=214)$ were determined to be high risk for a sexually transmitted infection. Overall, Clinic 1 was found to have a younger patient population ( $<5$ years old: $32.4 \%$ at Clinic 1 vs $9.5 \%$ at Clinic 2 ) and more likely to self-identify as black, non-Hispanic (70.9\% at Clinic 1 vs $13.61 \%$ at Clinic 2) (Table 1).

When the results were stratified by clinic, only Clinic 1 showed the following statistically significant increases after the intervention: PPSV23 for highrisk 19-64-year-olds (from $36.4 \%$ [ $\mathrm{n}=154]$ to $47.8 \%$ $[\mathrm{n}=136] ; \mathrm{P}=0.049)$; Hib vaccine for 2-month-to-5year-olds (from 91.1\% [ $\mathrm{n}=112]$ to $97.3 \%[\mathrm{n}=111]$; $\mathrm{P}=0.048$ ); and MenACWY for 13-18-year-olds (from $85.2 \%$ [ $\mathrm{n}=27]$ to $100 \%$ [ $\mathrm{n}=26] ; \mathrm{P}=0.042$ ) (Tables 2 and $3)$. When the results from the two clinics were pooled, HPV immunization rates for 13-18-year-olds also were increased (from $90.7 \%[\mathrm{n}=54]$ to $100 \%[\mathrm{n}=45]$; $\mathrm{P}=0.036$ ). Furthermore, rates at both studied clinics were higher across the board in comparison to national, state, and city data (Tables 2 and 3)., 22,23

Refugee patients ( $\mathrm{n}=171)$ comprised $10.1 \%$ of the total study population. As shown in Table 4, no increases were seen in vaccination rates within this subcohort, and a significant decrease was seen for receiving the second dose of the measles, mumps, and rubella vaccine (MMR\#2).

\section{DISCUSSION}

Our study found that a lecture-based educational intervention for nurses and MAs did not significantly increase immunization rates at both family medicine clinics. While Clinic 1 did show some statistically significant increases postintervention, Clinic 2 did not. This may be due to the attendance rate of nurses and MAs at Clinic 1 (100\% of participants attended all 3 sessions) being dramatically higher than the attendance rate at Clinic 2 (38\% of participants attended all 3 sessions).

Table 1. Patient Demographics ( $N=1689)$

\begin{tabular}{|c|c|c|c|c|c|c|}
\hline \multirow[b]{2}{*}{ Demographic } & \multicolumn{3}{|c|}{ Clinic 1} & \multicolumn{3}{|c|}{ Clinic 2} \\
\hline & $\begin{array}{c}\text { Pre } \\
n=449\end{array}$ & $\begin{array}{c}\text { Post } \\
n=417\end{array}$ & $\begin{array}{c}\text { Total } \\
\mathrm{n}=866\end{array}$ & $\begin{array}{c}\text { Pre } \\
n=423\end{array}$ & $\begin{array}{c}\text { Post } \\
n=400\end{array}$ & $\begin{array}{c}\text { Total } \\
\mathrm{n}=823\end{array}$ \\
\hline \multicolumn{7}{|l|}{ Age, n (\%) } \\
\hline $0-4.99$ years & $148(33.0 \%)$ & $133(31.9 \%)$ & $281(32.4 \%)$ & $41(9.7 \%)$ & $37(9.3 \%)$ & $78(9.5 \%)$ \\
\hline $5-18.99$ years & $52(11.6 \%)$ & $53(12.7 \%)$ & $105(12.0 \%)$ & $66(15.6 \%)$ & $54(13.5 \%)$ & $120(14.6 \%)$ \\
\hline 19-64.99 years & $221(49.2 \%)$ & $213(51.1 \%)$ & $434(50.1 \%)$ & $251(59.3 \%)$ & $244(61.0 \%)$ & $495(60.6 \%)$ \\
\hline$>65$ years & $28(6.2 \%)$ & $18(4.3 \%)$ & $46(5.3 \%)$ & $65(15.4 \%)$ & $65(16.3 \%)$ & $130(15.8 \%)$ \\
\hline \multicolumn{7}{|l|}{ Sex, n (\%) } \\
\hline Male & $182(40.5 \%)$ & $159(37.9 \%)$ & $341(39.4 \%)$ & $173(40.9 \%)$ & $150(37.5 \%)$ & $323(39.3 \%)$ \\
\hline Female & $267(59.5 \%)$ & $258(61.6 \%)$ & $525(60.6 \%)$ & $250(59.1 \%)$ & $250(62.5 \%)$ & $500(60.8 \%)$ \\
\hline \multicolumn{7}{|l|}{ Race/Ethnicity, n (\%) } \\
\hline White, non-Hispanic & $42(9.4 \%)$ & $49(11.8 \%)$ & $91(10.5 \%)$ & $136(32.2 \%)$ & $105(26.3 \%)$ & $241(29.3 \%)$ \\
\hline Hispanic & $41(9.1 \%)$ & $39(9.4 \%)$ & $80(9.2 \%)$ & $153(36.2 \%)$ & $162(40.5 \%)$ & $315(38.3 \%)$ \\
\hline Black, non-Hispanic & $322(71.7 \%)$ & $292(70.0 \%)$ & $614(70.9 \%)$ & $55(13.0 \%)$ & $57(14.3 \%)$ & $112(13.6 \%)$ \\
\hline Asian & $30(6.7 \%)$ & $27(6.5 \%)$ & $57(6.6 \%)$ & $44(10.4 \%)$ & $43(10.8 \%)$ & $87(10.6 \%)$ \\
\hline Mixed/Other/Unknown & $14(3.1 \%)$ & $10(2.4 \%)$ & $24(2.8 \%)$ & $35(8.3 \%)$ & $33(8.3 \%)$ & $68(8.3 \%)$ \\
\hline \multicolumn{7}{|l|}{ Refugee?, n (\%) } \\
\hline Yes & $36(8.0 \%)$ & $28(6.7 \%)$ & $64(7.4 \%)$ & $60(14.2 \%)$ & $47(11.8 \%)$ & $107(13.0 \%)$ \\
\hline No & $413(92.0 \%)$ & 389 (93.3\%) & $802(92.6 \%)$ & $363(85.8 \%)$ & $353(88.3 \%)$ & $716(87.0 \%)$ \\
\hline
\end{tabular}

Post, postintervention; Pre, preintervention; Total, pre- and postintervention combined. 
Table 2. Adult Risk-Based Vaccine Rates Compared to National, State, and City Rates and Further Stratified by Clinic ${ }^{a}$

\begin{tabular}{|c|c|c|c|c|c|c|c|c|c|c|}
\hline & \multirow[b]{2}{*}{ Pre } & \multirow[b]{2}{*}{ Post } & \multirow[b]{2}{*}{$P$} & \multirow[b]{2}{*}{$\begin{array}{c}\text { National } \\
\text { Data }^{1}\end{array}$} & \multirow[b]{2}{*}{$\begin{array}{l}\text { State } \\
\text { Data }^{22}\end{array}$} & \multirow[b]{2}{*}{$\begin{array}{c}\text { City } \\
\text { Data }^{22}\end{array}$} & \multicolumn{2}{|l|}{ Clinic 1} & \multicolumn{2}{|l|}{ Clinic 2} \\
\hline & & & & & & & $\begin{array}{l}\text { Pre / Post } \\
\text { Up-to-Date }\end{array}$ & $P$ & $\begin{array}{l}\text { Pre / Post } \\
\text { Up-to-Date }\end{array}$ & $P$ \\
\hline \multicolumn{11}{|l|}{ PPSV23 indications } \\
\hline 19-64 y/o at high risk & $\begin{array}{c}42.1 \% \\
(n=309)\end{array}$ & $\begin{array}{c}46.6 \% \\
(n=296)\end{array}$ & 0.261 & $23.0 \%$ & - & - & $\begin{array}{c}36.4 \%(n=154) / \\
47.8 \%(n=136)\end{array}$ & 0.049 & $\begin{array}{c}47.7 \%(n=155) / \\
45.6 \%(n=160)\end{array}$ & 0.708 \\
\hline$>65 \mathrm{y} / \mathrm{o}$ & $\begin{array}{l}83.3 \% \\
(n=90)\end{array}$ & $\begin{array}{l}92.1 \% \\
(n=76)\end{array}$ & 0.091 & $63.6 \%$ & $51.5 \%$ & $43.5 \%$ & $\begin{array}{l}76.9 \%(n=26) / \\
87.5 \%(n=16)\end{array}$ & 0.409 & $\begin{array}{c}85.9 \%(n=64) / \\
93.3 \%(n=60)\end{array}$ & 0.182 \\
\hline Diabetics & $\begin{array}{c}68.6 \% \\
(n=137)\end{array}$ & $\begin{array}{c}76.4 \% \\
(n=148)\end{array}$ & 0.144 & - & - & - & $\begin{array}{l}57.7 \%(n=52) / \\
71.2 \%(n=52)\end{array}$ & 0.155 & $\begin{array}{l}75.3 \%(n=85) / \\
80.0 \%(n=95)\end{array}$ & 0.451 \\
\hline Heart/Lung disease & $\begin{array}{c}50.0 \% \\
(n=318)\end{array}$ & $\begin{array}{c}52.5 \% \\
(n=301)\end{array}$ & 0.536 & - & - & - & $\begin{array}{c}43.0 \%(n=151) / \\
50.8 \%(n=128)\end{array}$ & 0.198 & $\begin{array}{c}55.8 \%(n=165) / \\
54.1 \%(n=172)\end{array}$ & 0.756 \\
\hline \multicolumn{11}{|l|}{ Hep B indications } \\
\hline Chronic liver disease & $\begin{array}{l}41.9 \% \\
(n=43)\end{array}$ & $\begin{array}{l}39.6 \% \\
(n=48)\end{array}$ & 0.827 & $27.4 \%$ & - & - & $\begin{array}{c}33.3 \%(n=21) / \\
30.0 \%(n=10)\end{array}$ & 0.859 & $\begin{array}{c}26.7 \%(n=15) / \\
35.3 \%(n=34)\end{array}$ & 0.562 \\
\hline Diabetic, $19-59$ y/o & $\begin{array}{l}37.3 \% \\
(n=75)\end{array}$ & $\begin{array}{l}29.9 \% \\
(n=87)\end{array}$ & 0.319 & $24.4 \%$ & - & - & $\begin{array}{l}28.1 \%(n=32) / \\
26.8 \%(n=41)\end{array}$ & 0.904 & $\begin{array}{c}44.2 \%(n=43) / \\
32.6 \%(n=46)\end{array}$ & 0.266 \\
\hline At risk for STI & $\begin{array}{l}54.5 \% \\
(n=88)\end{array}$ & $\begin{array}{c}46.0 \% \\
(n=126)\end{array}$ & 0.222 & - & - & - & $\begin{array}{c}52.5 \%(n=61) / \\
50.7 \%(n=69)\end{array}$ & 0.845 & $\begin{array}{c}54.2 \%(n=24) / \\
38.2 \%(n=55)\end{array}$ & 0.192 \\
\hline \multicolumn{11}{|l|}{ Tdap indications } \\
\hline $19-64$ y/o & $\begin{array}{c}87.4 \% \\
(n=469)\end{array}$ & $\begin{array}{c}88.4 \% \\
(n=457)\end{array}$ & 0.647 & - & $62.0 \%$ & $52.0 \%$ & $\begin{array}{c}94.1 \%(n=221) / \\
94.4 \%(n=213)\end{array}$ & 0.912 & $\begin{array}{c}81.5 \%(n=248) / \\
83.2 \%(n=244)\end{array}$ & 0.613 \\
\hline Pregnant $^{\mathrm{b}}$ & $\begin{array}{l}100 \% \\
(n=9)\end{array}$ & $\begin{array}{l}90.9 \% \\
(n=11)\end{array}$ & 0.380 & - & - & - & $\begin{array}{c}100 \%(n=4) / \\
100 \%(n=8)\end{array}$ & 1.000 & $\begin{array}{c}100 \%(n=6) / \\
66 \%(n=3)\end{array}$ & 1.000 \\
\hline \multicolumn{11}{|l|}{ Other: indications } \\
\hline Нер A: >19 y/o & $\begin{array}{l}16.3 \% \\
(n=43)\end{array}$ & $\begin{array}{l}17.0 \% \\
(n=53)\end{array}$ & 0.928 & - & - & - & $\begin{array}{c}21.7 \%(n=23) / \\
20.0 \%(n=15)\end{array}$ & 0.901 & $\begin{array}{c}10.0 \%(n=20) / \\
15.8 \%(n=38)\end{array}$ & 0.552 \\
\hline $\mathrm{Td}:>19 \mathrm{y} / \mathrm{o}$ & $\begin{array}{c}84.7 \% \\
(n=562)\end{array}$ & $\begin{array}{c}87.0 \% \\
(n=540)\end{array}$ & 0.266 & $61.6 \%$ & - & - & $\begin{array}{c}90.8 \%(n=249) / \\
93.5 \%(n=231)\end{array}$ & 0.267 & $\begin{array}{c}79.9 \%(n=313) / \\
82.2 \%(n=309)\end{array}$ & 0.460 \\
\hline MMR: >19 y/o & $\begin{array}{c}41.6 \% \\
(n=353)\end{array}$ & $\begin{array}{c}47.0 \% \\
(n=353)\end{array}$ & 0.150 & - & - & - & $\begin{array}{c}42.8 \%(n=166) / \\
50.3 \%(n=173)\end{array}$ & 0.166 & $\begin{array}{c}40.6 \%(n=187) / \\
43.9 \%(n=180)\end{array}$ & 0.530 \\
\hline PCV13: >65 y/o & $\begin{array}{l}83.7 \% \\
(n=92)\end{array}$ & $\begin{array}{l}90.1 \% \\
(n=81)\end{array}$ & 0.216 & $49.8 \%$ & $49.8 \%$ & $35.4 \%$ & $\begin{array}{c}74.1 \%(n=27) / \\
94.1 \%(n=17)\end{array}$ & 0.097 & $\begin{array}{c}87.7 \%(n=65) / \\
89.1 \%(n=64)\end{array}$ & 0.810 \\
\hline
\end{tabular}

Post, postintervention; Pre, preintervention; STI, sexually transmitted infection; y/o, years old. See Box 1 for vaccine abbreviations. ${ }^{a}$ Results only for patients $>19$ years of age $(n=1105)$.

${ }^{b}$ Pregnant refers only to patients at 27-36 weeks gestation.

Immunization rates in Milwaukee, Wisconsin, where the study was performed, are lower than state and national averages. However, when compared to nationwide, Wisconsin-wide, and Milwaukee-wide rates, immunization rates at the two clinics making up our study setting were much higher at baseline. These already high rates may have contributed to the lack of response to the intervention.
Some barriers to vaccination from the patient's perspective include lack of caregiver knowledge, mistrust of vaccines, and caregiver's perceived barriers to accessing the health care system. ${ }^{24-26}$ Barriers from the clinician's perspective include concerns for cost, insurance coverage, and incorrect assumptions of immunity. ${ }^{27}$ As an example, Tdap vaccine is not covered by Medicare Part B unless it is part of the treatment for 
Table 3. Pediatric Vaccine Rates Compared to National, State, and City Rates and Further Stratified by Clinic ${ }^{a}$

\begin{tabular}{|c|c|c|c|c|c|c|c|c|c|c|}
\hline \multirow[b]{2}{*}{$\begin{array}{l}\text { Vaccine (Patient } \\
\text { Age Range) }\end{array}$} & \multirow[b]{2}{*}{ Pre } & \multirow[b]{2}{*}{ Post } & \multirow[b]{2}{*}{$P$} & \multirow[b]{2}{*}{$\begin{array}{c}\text { National } \\
\text { Data }^{1}\end{array}$} & \multirow[b]{2}{*}{$\begin{array}{c}\text { State } \\
\text { Data }^{22}\end{array}$} & \multirow[b]{2}{*}{$\begin{array}{c}\text { City } \\
\text { Data }^{22}\end{array}$} & \multicolumn{2}{|l|}{ Clinic 1} & \multicolumn{2}{|l|}{ Clinic 2} \\
\hline & & & & & & & $\begin{array}{l}\text { Pre / Post } \\
\text { Up-to-Date }\end{array}$ & $P$ & $\begin{array}{l}\text { Pre / Post } \\
\text { Up-to-Date }\end{array}$ & $P$ \\
\hline $\begin{array}{l}\text { Polio } \\
\text { (2 months-18 years) }\end{array}$ & $\begin{array}{c}97.4 \% \\
(n=265)\end{array}$ & $\begin{array}{c}98.0 \% \\
(n=244)\end{array}$ & 0.661 & - & - & - & $\begin{array}{c}97.6 \%(n=164) / \\
98.2 \%(n=164)\end{array}$ & 0.703 & $\begin{array}{c}97.0 \%(n=101) / \\
97.5 \%(n=80)\end{array}$ & 0.849 \\
\hline $\begin{array}{l}\text { DTaP/Tdap } \\
\text { (2 months-18 years) }\end{array}$ & $\begin{array}{c}96.9 \% \\
(n=266)\end{array}$ & $\begin{array}{c}97.1 \% \\
(n=245)\end{array}$ & 0.434 & - & - & - & $\begin{array}{c}95.7 \%(n=164) / \\
98.2 \%(n=164)\end{array}$ & 0.200 & $\begin{array}{c}96.1 \%(n=102) / \\
95.1 \%(n=81)\end{array}$ & 0.740 \\
\hline $\begin{array}{l}\text { Hib } \\
\text { ( } 2 \text { months }-5 \text { years })\end{array}$ & $\begin{array}{c}91.9 \% \\
(n=149)\end{array}$ & $\begin{array}{c}97.1 \% \\
(n=138)\end{array}$ & 0.057 & - & - & - & $\begin{array}{c}91.1 \%(n=112) / \\
97.3 \%(n=111)\end{array}$ & 0.048 & $\begin{array}{c}94.6 \%(n=37) / \\
96.3 \%(n=27)\end{array}$ & 0.755 \\
\hline $\begin{array}{l}\text { Hep A } \\
\text { (1-18 years) }\end{array}$ & $\begin{array}{c}94.4 \% \\
(n=196)\end{array}$ & $\begin{array}{c}95.2 \% \\
(n=187)\end{array}$ & 0.726 & - & - & - & $\begin{array}{c}92.5 \%(n=107) / \\
96.6 \%(n=116)\end{array}$ & 0.184 & $\begin{array}{c}96.6 \%(n=89) / \\
93.0 \%(n=71)\end{array}$ & 0.293 \\
\hline $\begin{array}{l}\text { Hep B } \\
\text { (0 months-18 years) }\end{array}$ & $\begin{array}{l}96.7 \% \\
(n=307)\end{array}$ & $\begin{array}{c}97.8 \% \\
(n=277)\end{array}$ & 0.421 & - & - & - & $\begin{array}{c}96.0 \%(n=200) / \\
97.3 \%(n=186)\end{array}$ & 0.477 & $\begin{array}{c}98.1 \%(n=107) / \\
98.9 \%(n=91)\end{array}$ & 0.660 \\
\hline $\begin{array}{l}\text { Rotavirus } \\
\text { (2-8 months) }\end{array}$ & $\begin{array}{l}94.5 \% \\
(n=55)\end{array}$ & $\begin{array}{l}86.4 \% \\
(n=44)\end{array}$ & 0.162 & - & - & - & $\begin{array}{c}95.3 \%(n=43) / \\
83.3 \%(n=36)\end{array}$ & 0.080 & $\begin{array}{c}91.7 \%(n=12) / \\
100 \%(n=8)\end{array}$ & 0.429 \\
\hline $\begin{array}{l}\text { MMR } \\
\text { (2-8 months) }\end{array}$ & $\begin{array}{c}97.4 \% \\
(n=194)\end{array}$ & $\begin{array}{c}97.3 \% \\
(n=186)\end{array}$ & 0.946 & - & $85.7 \%^{c}$ & $85.8 \%^{c}$ & $\begin{array}{c}95.2 \%(n=105) / \\
97.4 \%(n=116)\end{array}$ & 0.389 & $\begin{array}{l}100 \%(n=89) / \\
97.1 \%(n=70)\end{array}$ & 0.110 \\
\hline $\begin{array}{l}\text { MenACWY } \\
\text { (13-18 years) }\end{array}$ & $\begin{array}{l}92.6 \% \\
(n=54)\end{array}$ & $\begin{array}{l}97.7 \% \\
(n=44)\end{array}$ & 0.255 & $82.2 \%^{d}$ & $70.9 \%$ & $68.6 \%$ & $\begin{array}{c}85.2 \%(n=27) / \\
100 \%(n=26)\end{array}$ & 0.042 & $\begin{array}{l}100 \%(n=27) / \\
94.4 \%(n=18)\end{array}$ & 0.225 \\
\hline $\begin{array}{l}\text { Varicella } \\
\text { (1-18 years) }\end{array}$ & $\begin{array}{c}97.1 \% \\
(n=138)\end{array}$ & $\begin{array}{c}96.8 \% \\
(n=186)\end{array}$ & 0.867 & - & $84.1 \%{ }^{c}$ & $84.8 \%^{c}$ & $\begin{array}{l}95.2 \%(n=83) / \\
96.6 \%(n=116)\end{array}$ & 0.629 & $\begin{array}{l}100 \%(n=55) / \\
97.1 \%(n=70)\end{array}$ & 0.209 \\
\hline $\begin{array}{l}\text { PCV13 } \\
(2 \text { months-18 years })^{b}\end{array}$ & $\begin{array}{l}89.3 \% \\
(n=149)\end{array}$ & $\begin{array}{l}89.1 \% \\
(n=138)\end{array}$ & 0.972 & - & - & - & $\begin{array}{c}87.5 \%(n=112) / \\
86.5 \%(n=111)\end{array}$ & 0.823 & $\begin{array}{c}94.6 \%(n=37) / \\
100 \%(n=27)\end{array}$ & 0.226 \\
\hline $\begin{array}{l}\text { HPV } \\
\text { (13-18 years) }\end{array}$ & $\begin{array}{l}90.7 \% \\
(n=54)\end{array}$ & $\begin{array}{l}100 \% \\
(n=45)\end{array}$ & 0.036 & $60.4 \%^{d}$ & $53.3 \%$ & $57.8 \%$ & $\begin{array}{c}92.6 \%(n=27) / \\
100 \%(n=26)\end{array}$ & 0.163 & $\begin{array}{c}88.9 \%(n=27) / \\
100 \%(n=19)\end{array}$ & 0.139 \\
\hline
\end{tabular}

Post, postintervention; Pre, preintervention. See Box 1 for vaccine abbreviations.

${ }^{a}$ Results only for patients $<19$ years of age $(n=584)$.

${ }^{b}$ Patients 5-18 years old who were indicated for the vaccine had a high-risk indication.

'Rate determined by 24 months of age.

${ }^{d}$ Rate for 13-17-year-olds.

an injury or direct exposure to the disease. ${ }^{28}$ The MMR vaccine rates for adults at the clinics studied were less than $50 \%$. MMR is possibly considered a low priority by clinicians because individuals born in 1957 or earlier $(60$ years old or older at the time of this study) are considered immune. ${ }^{15}$ Assuming that after 2022, all 19-64-yearolds will be eligible for MMR, this educational point may need to be addressed in future interventions.

No increases in vaccination rates were observed among refugee patients. Based on their age and risk factors, refugee patients must be vaccinated with the following immunizations - Polio, DTaP/DT/Td/Tdap, Hib, Hep A, Hep B, Rotavirus, MMR, Meningococcal, Varicella, Pneumococcal, and Influenza - before they can submit a permanent residency application. ${ }^{29}$ These requirements could explain the clinics' high vaccination rates for refugee patients, which made it harder to improve these already high rates. However, PPSV23 immunization rates for refugees still require much improvement. Potential explanations for the worsened MMR\#2 immunization rate postintervention include patient refusal, shortage of the vaccine, or the vaccine just being missed by clinic staff.

\section{Limitations}

This study was subject to several limitations. Because Wisconsin's immunization registry came online in 2000 , information in the registry about vaccines administered before 2000 is limited. While staff at local 
Table 4. Refugee Vaccine Rates ${ }^{a}$

\begin{tabular}{lccc}
\hline Vaccine & Preintervention & Postintervention & $\boldsymbol{P}$ \\
\hline Polio & $90.9 \%(n=33)$ & $100 \%(n=36)$ & 0.066 \\
$\begin{array}{l}\text { Tdap adult } \\
(>19 \text { y/o })\end{array}$ & $93.1 \%(n=58)$ & $89.2 \%(n=37)$ & 0.508 \\
$\begin{array}{l}\text { DTaP/Tdap } \\
(<19 \text { y/o })\end{array}$ & $91.2 \%(n=34)$ & $100 \%(n=36)$ & 0.070 \\
Td & $96.5 \%(n=57)$ & $91.9 \%(n=37)$ & 0.337 \\
Hib & $95.7 \%(n=23)$ & $100 \%(n=24)$ & 0.312 \\
Hep A & $73.5 \%(n=34)$ & $74.2 \%(n=31)$ & 0.952 \\
Hep B & $84.6 \%(n=52)$ & $88.0 \%(n=50)$ & 0.624 \\
Rotavirus & $100 \%(n=6)$ & $100 \%(n=8)$ & - \\
MMR & $92.1 \%(n=63)$ & $94.1 \%(n=51)$ & 0.673 \\
MMR \#2 & $100 \%(n=23)$ & $87.0 \%(n=23)$ & 0.036 \\
Meningococcal & $100 \%(n=4)$ & $80.0 \%(n=5)$ & 0.407 \\
Varicella & $77.5 \%(n=40)$ & $82.6 \%(n=46)$ & 0.558 \\
PCV13 & $86.2 \%(n=29)$ & $92.0 \%(n=25)$ & 0.509 \\
PPSV23 & $37.9 \%(n=29)$ & $53.3 \%(n=15)$ & 0.340 \\
\hline
\end{tabular}

y/o, years old. See Box 1 for vaccine abbreviations.

${ }^{a}$ Results only for refugee patients $(n=171)$.

health departments routinely enter into the registry records of vaccinations administered before 2000, staff at primary care clinics or pharmacies do not always. ${ }^{30}$ Furthermore, vaccines administered in different states rarely make it into the registry. As a result, compared to children, adults have less complete documentation of vaccinations in the registry. Therefore, the up-to-date vaccination rates in this study may be lower than actual rates. On the other hand, because the postintervention period spanned the flu season, the reported vaccination rates may have skewed higher due to more focus being placed on immunization when visits happen during the flu season. Lastly, this study does not have the capacity to analyze many of the systemic factors that contribute to low immunization rates.

\section{CONCLUSIONS}

A short-term educational program for nurses and medical assistants at two family medicine residency clinics did not have a significant effect in improving most immunizations rates, likely due to the clinics' already high age- and risk-based immunization rates. Despite the fact that, on average, these rates were higher than national, Wisconsin, and Milwaukee rates, there is still opportunity for improvement.

While a multidisciplinary team approach to this problem is required, the development of algorithms in electronic health records to generate patient-specific, risk-based vaccine recommendations at the time of a clinic visit, in addition to the age-based forecasts available from state immunization registries, would be helpful. However, even these interventions will have difficulty improving the population's overall immunization rates if systemic factors affecting vulnerable populations are not addressed. Therefore, future interventions must address systemic factors in Milwaukee, like lack of access to care, if the city is to reach Healthy People 2020's immunization goals.

\section{Patient-Friendly Recap}

- Compared to the general U.S. population, immunization rates in cities often lag.

- The authors targeted an educational lecture series to nurses and medical assistants in the hope of improving vaccination rates at two urban clinics.

-While the education did not result in substantial increases in vaccinations, this may have been in part due to unusually high baseline rates at the studied clinics.

- The authors conclude that future attempts to improve immunization rates should go beyond education and address systemic factors such as access to care.

\section{Author Contributions}

Study design: Yacoob, Kotovicz, Stanley, Hunter. Data acquisition or analysis: Yacoob, Cook, Kotovicz, Stanley, Hunter. Manuscript drafting: Yacoob, Kotovicz, Kram, Klumph, Stanley, Hunter, Baumgardner. Critical revision: Kotovicz, Kram, Klumph, Hunter, Baumgardner.

\section{Conflicts of Interest}

None.

\section{Funding Sources}

This quality improvement project was funded in part by a micro grant from University of Wisconsin School of Medicine and Public Health's Department of Family Medicine and Community Health as well as funding from the Aurora UW Medical Group Faculty Pilot Fund. 


\section{References}

1. Williams WW, Lu PJ, O'Halloran A, et al. Surveillance of vaccination coverage among adult populations - United States, 2015. MMWR Surveill Summ. 2017;66:1-28. CrossRef

2. Centers for Disease Control and Prevention. Estimates of deaths associated with seasonal influenza - United States, 1976-2007. MMWR Morb Mortal Wkly Rep. 2010;59:1057-62.

3. Centers for Disease Control and Prevention. Viral hepatitis surveillance: United States, 2013. https:/www.cdc.gov/hepatitis/ statistics/2013surveillance/pdfs/2013hepsurveillancerpt.pdf. Accessed August 12, 2018.

4. National Vaccine Advisory Committee. Enhancing the work of the Department of Health and Human Services national vaccine program in global immunization: recommendations of the National Vaccine Advisory Committee. Public Health Rep. 2014;129 Suppl 3:12-85.

5. Jacobson Vann JC, Jacobson RM, Coyne-Beasley T, AsafuAdjei JK, Szilagyi PG. Patient reminder and recall interventions to improve immunization rates. Cochrane Database Syst Rev. 2018;1:CD003941. $\underline{\text { CrossRef }}$

6. Smith JG, Metzger NL. Evaluation of pneumococcal vaccination rates after vaccine protocol changes and nurse education in a tertiary care teaching hospital. J Manag Care Pharm. 2011;17:701-8. CrossRef

7. Ford JA, Wong G, Jones AP, Steel N. Access to primary care for socioeconomically disadvantaged older people in rural areas: a realist review. BMJ Open. 2016;6(5):e010652. CrossRef

8. Agency for Healthcare Research and Quality. 2017 National Healthcare Quality and Disparities Report. Last reviewed 2019 Jul. http://www.ahrq.gov/research/findings/nhqrdr/nhqdr17/ index.html. Accessed November 1, 2018.

9. Beasley JW, Hankey TH, Erickson R, et al. How many problems do family physicians manage at each encounter? A WReN Study. Ann Fam Med. 2004;2:405-10. CrossRef

10. Roemheld-Hamm B, Isaacson N, Winston C, Scott J, Hudson $\mathrm{S}$, Crabtree B. Influenza immunization disparities in primary care offices - a comparative case study. J Health Care Poor Underserved. 2008;19:1248-57. CrossRef

11. Baker AN, Parsons M, Donnelly SM, et al. Improving colon cancer screening rates in primary care: a pilot study emphasizing the role of the medical assistant. Qual Saf Health Care. 2009;18:355-9. CrossRef

12. Mohler PJ. Enhancing compliance with screening mammography recommendations: a clinical trial in a primary care office. Fam Med. 1995;27:117-21.

13. Alexander KE, Brijnath B, Mazza D. The challenges of trying to increase preventive healthcare for children in general practice: results of a feasibility study. BMC Fam Pract. 2015;16:94. CrossRef

14. Thomas RE, Lorenzetti DL. Interventions to increase influenza vaccination rates of those 60 years and older in the community. Cochrane Database Syst Rev. 2018;5:CD005188. CrossRef

15. Kim DK, Riley LE, Harriman KH, Hunter P, Bridges CB. Advisory Committee on Immunization Practices recommended immunization schedule for adults aged 19 years or older - United States, 2017. MMWR Morb Mortal Wkly Rep. 2017;66:136-8. CrossRef

16. Robinson CL, Romero JR, Kempe A, Pellegrini C; Advisory Committee on Immunization Practices Child/Adolescent Immunization Work Group. Advisory Committee on Immunization Practices recommended immunization schedule for children and adolescents aged 18 years or younger - United States, 2017. MMWR Morb Mortal Wkly Rep. 2017;66:134-5. CrossRef
17. Centers for Disease Control and Prevention; Advisory Committee on Immunization Practices. Updated recommendations for prevention of invasive pneumococcal disease among adults using the 23-valent pneumococcal polysaccharide vaccine (PPSV23). MMWR Morb Mortal Wkly Rep. 2010;59:1102-6.

18. Immunization Action Coalition. Ask the experts: pneumococcal vaccines (PCV13 and PPSV23). Last updated 2019 Jul 2. http:/www.immunize.org/askexperts/experts_pneumococcal_ vaccines.asp. Accessed May 16, 2018.

19. National Institutes of Health, National Institute on Alcohol Abuse and Alcoholism. Helping patients who drink too much: a clinician's guide. Updated in 2005. https://www.integration. samhsa.gov/clinical-practice/Helping_Patients_Who_Drink_ Too_Much.pdf. Accessed August 13, 2018.

20. American Academy of Pediatrics. Documenting parental refusal to have their children vaccinated. Published in 2013. https://www.aap.org/en-us/Documents/immunization_ refusaltovaccinate.pdf. Accessed August 4, 2017.

21. Centers for Disease Control and Prevention. Pneumococcal vaccine timing for adults. Released 2015 Nov 30. https://www. cdc.gov/vaccines/vpd/pneumo/downloads/pneumo-vaccinetiming.pdf. Accessed August 13, 2018.

22. Wisconsin Department of Health Services. Immunization rate data. Last revised 2019 Jul 24. https:/www.dhs.wisconsin.gov/ immunization/data.htm. Accessed May 19, 2018.

23. Walker TY, Elam-Evans LD, Singleton JA, et al. National, Regional, State, and Selected Local Area Vaccination Coverage Among Adolescents Aged 13-17 Years - United States, 2016. MMWR Morb Mortal Wkly Rep. 2017;66:874-82. CrossRef

24. Weiner JL, Fisher AM, Nowak GJ, Basket MM, Gellin BG. Childhood immunizations: first-time expectant mothers' knowledge, beliefs, intentions, and behaviors. Am J Prev Med. 2015;49:S426-34. CrossRef

25. Katz I, Bogart LM, Fu CM, et al. Barriers to HPV immunization among blacks and latinos: a qualitative analysis of caregivers, adolescents, and providers. BMC Public Health. 2016;16(1):874. CrossRef

26. Niederhauser VP, Lindley LC, Farabollini B. Changes in parental barriers to immunizations. Public Health Nurs. 2018;35:379-85. CrossRef

27. Hurley LP, Lindley MC, Allison MA, et al. Primary care physicians' perspective on financial issues and adult immunization in the era of the Affordable Care Act. Vaccine. 2017;35:647-54. CrossRef

28. Centers for Medicare \& Medicaid Services. Medicare Learning Network fact sheet: Medicare Part D vaccines. Updated 2019 Jun. https://www.cms.gov/Outreach-and-Education/MedicareLearning-Network-MLN/MLNProducts/Downloads/VaccinesPart-D-Factsheet-ICN908764.pdf. Accessed August 13, 2018.

29. U.S. Department of Homeland Security, U.S. Citizenship and Immigration Services. Vaccination requirements. Last updated 2018 Mar 8. https://www.uscis.gov/news/questions-andanswers/vaccination-requirements. Accessed July 3, 2017.

30. Koepke R, Petit AB, Ayele RA, et al. Completeness and accuracy of the Wisconsin Immunization Registry: an evaluation coinciding with the beginning of meaningful use. J Public Health Manag Pract. 2015;21:273-81. CrossRef

(C) 2020 Aurora Health Care, Inc. 


\begin{tabular}{|c|c|}
\hline \multicolumn{2}{|c|}{ Appendix A: Definitions for High-Risk Populations } \\
\hline Indication & Definition \\
\hline Kidney failure & Estimated glomerular filtration rate at time of visit was $<30 \mathrm{ml} / \mathrm{min}$ \\
\hline Cardiac indication & $\begin{array}{l}\text { If patient had any of the following: } \\
\text { - Any structural abnormality of the heart } \\
\text { - Cardiomyopathy excluding hypertension } \\
\text { - Any known electrocardiogram abnormalities (excluding bradycardia and tachycardia) }\end{array}$ \\
\hline Pulmonary indication & $\begin{array}{l}\text { If patient had any of the following: }:^{17} \\
\text { - Significant structural abnormality of the lung } \\
\text { - Chronic lung disease (for asthma to be considered as a chronic lung disease, patient } \\
\text { had to have had a prescription for a controller medication) } \\
\text { - Current smoker (patients were not considered to be smokers if they used cannabis or } \\
\text { smokeless tobacco }{ }^{18} \text { ) }\end{array}$ \\
\hline Alcohol abuse disorder & $\begin{array}{l}\text { Any patient who had a history of alcohol abuse or exceeded national drinking guidelines } \\
\text { was determined to have alcohol abuse disorder }{ }^{19}\end{array}$ \\
\hline Intravenous drug use & Any patient who had a past history of intravenous drug use \\
\hline Liver disease & $\begin{array}{l}\text { If patient had any of the following: } \\
\text { - Cirrhosis } \\
\text { - Fatty liver disease } \\
\text { - Alcoholic liver disease } \\
\text { - Alcoholic hepatitis } \\
\text { - Aspartate aminotransferase or alanine aminotransferase level >2 times the upper limit of } \\
\text { normal (liver function tests for every patient with available results were analyzed) } \\
\text { - Nonalcoholic steatohepatitis } \\
\text { - Chronic hepatitis B } \\
\text { - Hepatitis C infection }\end{array}$ \\
\hline $\begin{array}{l}\text { High risk for sexually } \\
\text { transmitted infection (STI) }\end{array}$ & $\begin{array}{l}\text { If patient had any of the following: } \\
\text { - Greater than } 1 \text { sexual partner in the past } 6 \text { months. } \\
\text { - Ever been diagnosed with an STI, including gonorrhea, chlamydia, high-risk human } \\
\text { papilloma virus, genital herpes, and/or human immunodeficiency virus } \\
\text { - History of sexual abuse, cervical cancer, and/or multiple sexual partners } \\
\text { - Patient concerned about having an STI or requesting STI screening }\end{array}$ \\
\hline Refugee & $\begin{array}{l}\text { If the patient's chart included any of the following informationa: } \\
\text { - Patient's primary or secondary language was listed as Karen, Burmese, Rohingya, } \\
\text { Arabic, Chin, Somali, or Malay } \\
\text { - Children who were born to refugee parents } \\
\text { - Patient's chart identified them as a refugee }\end{array}$ \\
\hline
\end{tabular}




\begin{tabular}{|c|c|c|}
\hline \multicolumn{3}{|c|}{ Appendix B: Quick Reference Guide ${ }^{a}$} \\
\hline Travelers & \multicolumn{2}{|c|}{$\begin{array}{l}\text { - Polio } \\
\text { - Hepatitis A } \\
\text { - Hepatitis B } \\
\text { - Measles, mumps, and rubella (ensure } 2 \text { doses received) } \\
\text { - Meningococcal }\end{array}$} \\
\hline $\begin{array}{l}\text { Asplenia (sickle cell } \\
\text { disease) }\end{array}$ & \multicolumn{2}{|l|}{$\begin{array}{l}\text { - Haemophilus influenzae type B } \\
\text { - Meningococcal } \\
\text { - Pneumococcal: PPSV23 + PCV13 }\end{array}$} \\
\hline $\begin{array}{l}\text { Immunocompromised } \\
\text { individuals }\end{array}$ & \multicolumn{2}{|c|}{$\begin{array}{l}\text { - Ex: HIV, chemotherapy, radiation therapy, high-dose steroids for }>14 \text { days } \\
\text { - Haemophilus influenzae type B } \\
\text { - Meningococcal } \\
\text { - Pneumococcal: PPSV23 + PCV13 }\end{array}$} \\
\hline $\begin{array}{l}\text { Men who have sex } \\
\text { with men }\end{array}$ & \multicolumn{2}{|l|}{$\begin{array}{l}- \text { Hepatitis A } \\
\text { - Hepatitis B }\end{array}$} \\
\hline $\begin{array}{l}\text { History of intravenous } \\
\text { drug use }\end{array}$ & \multicolumn{2}{|l|}{$\begin{array}{l}\text { - Hepatitis A } \\
\text { - Hepatitis B }\end{array}$} \\
\hline $\begin{array}{l}\text { Vaccines needed by } \\
\text { refugees }\end{array}$ & $\begin{array}{l}\text { - Inactivated polio vaccine } \\
\text { - Tetanus, diphtheria, and pertussis } \\
\text { - Haemophilus influenzae type B } \\
\text { - Hepatitis A } \\
\text { - Hepatitis B } \\
\text { - Rotavirus }\end{array}$ & $\begin{array}{l}\text { - Measles, mumps, and rubella } \\
\text { - Meningococcal } \\
\text { - Varicella } \\
\text { - Pneumococcal } \\
\text { - Seasonal influenza }\end{array}$ \\
\hline HIV & \multicolumn{2}{|l|}{$\begin{array}{l}\text { - Haemophilus influenzae type B } \\
\text { - Hepatitis B } \\
\text { - Pneumococcal: PPSV23 + PCV13 }\end{array}$} \\
\hline Kidney failure & \multicolumn{2}{|l|}{$\begin{array}{l}\text { - Hepatitis B } \\
\text { - Pneumococcal: PPSV23 + PCV13 }\end{array}$} \\
\hline Diabetes & \multicolumn{2}{|c|}{$\begin{array}{l}\text { - Hepatitis B } \\
\text { - Pneumococcal: } \\
\quad \text { - Single dose of PPSV23 } \\
\text { - If less than 18, then a dose of PCV13 also }\end{array}$} \\
\hline Multiple sex partners & \multicolumn{2}{|l|}{ - Hepatitis B } \\
\hline College students & \multicolumn{2}{|c|}{$\begin{array}{l}\text { - Measles, mumps, and rubella (ensure patient has received } 2 \text { doses) } \\
\text { - Meningococcal }\end{array}$} \\
\hline $\begin{array}{l}\text { Special points and } \\
\text { resources }\end{array}$ & \multicolumn{2}{|c|}{$\begin{array}{l}\text { - Any adult born in } 1957 \text { or later must have MMR vaccine documented in chart. If no } \\
\text { documentation of MMR, then vaccinate. } \\
\text { - Look up vaccines that may be needed: } \mathrm{https://www2a.cdc.gov/nip/adultimmsched/default.asp} \\
\text { - Pneumococcal recommendations for adults: https://www.cdc.gov/vaccines/vpd/pneumo/ } \\
\text { downloads/pneumo-vaccine-timing.pdf }\end{array}$} \\
\hline \multicolumn{3}{|c|}{${ }^{a}$ For vaccine abbreviations, see Box 1 on p. 49.} \\
\hline
\end{tabular}

